\title{
Intestinal duplication
}

\author{
H. K. Ramakrishna
}

Received: 3 October 2008 / Accepted: 28 October 2008

\begin{abstract}
Gastrointestinal duplications are rare but interesting clinical entities. They have a varied presentation, with most of them showing up in paediatric population. Clinical features may vary from asymptomatic abdominal masses to bowel obstruction or perforation. This review traces the embryological origin and describes the anatomical types of duplications. An outline of the principles of management is described.
\end{abstract}

Keywords Intestinal duplication - Duplication cyst . Enteric cysts.

H. K. Ramakrishna $(\bowtie)$

Lakshmi Surgical and Endoscopic Unit, New Brdige Road, Bhadravati - 577 301, India

E mail: swarama@hotmail.com; swarama@gmail.com

\section{Introduction}

Duplications are rare congenital anomalies which can occur anywhere in GIT from mouth to anus. Most of the literature search gives only case reports. As Gray AW remarked "gastrointestinal tract is a fertile field for various and curious congenital malformations which are fascinating to study for both surgeon and pathologist" [1].

\section{Embryology}

There are many theories for development of duplication. According to one of the theories it is due to persistence of outpouchings of the developing intestines which occur between 6 to 8 weeks of intrauterine life. According to another theory, there is an attachment between the gut endoderm and the neural tube ectoderm, which separates normally in the fourth week of intrauterine development. If this fails, gut will be pulled towards developing vertebral bodies at the site of attachment which results in traction and development of tubular outpouchings, the duplications. If the attachment gets detached at both ends, a duplication cyst results. Theory of aberrant recanalisation is put forward by Bremer in 1944 [2], where he postulates during vacuoles formation and their coalescence to form a single channel, instead of one, two channels form, which may or may not communicate with each other.

\section{Types}

Most commonly duplications are classified as two types, the tubular and the cystic varieties. Tubular duplications tend to have communication with the gut lumen commonly at the caudal end but can have communication at both the ends. Cystic type is commoner (65\%). Cystic ones are completely separate from the intestine. Li et al [3] have classified small intestinal duplications depending upon the vascular pattern. 
Type 1 or Parallel type (74.6\%): Duplication is on one of the leaves of the mesentery. Straight artery of the duplication is separate from the straight artery of the bowel.

Type 1a: Duplication has a separate mesentery.

Type 1b: Duplication shares common mesentery with the bowel.

Type 1c: Duplication shares common muscular coat with the bowel.

Type 2 or Intra mesenteric type (24.4\%): Duplication is located between the two leaves of the mesentery. Straight arteries pass over both the surfaces of the duplication to reach the bowel.

Type 2a: Duplication separate from the bowel.

Type 2b: Duplication shares common muscular coat with the bowel.

They found that vertebral defects were found more commonly associated with type 2 variety $(91.6 \%)$ than with type 1 variety (5.5\%). They claim the study of vascular pattern allows excision of the duplication in small bowel without the need for resection of the small bowel as is commonly practiced. In type 1 , mesentery divided from the straight artery and contra lateral artery is kept intact. In type 2 , the small branches of the straight arteries are to be divided and the duplication is enucleated. Thus, resection of the bowel avoided resulting in decreased morbidity and operating time.

\section{Site}

Majority of the duplications occur in small intestines, especially in the ileum. They lie towards the mesenteric side of the intestine. Same patient may have more than one lesion.

Some of them may be confined to the thorax (21/ 101) [4]. Rarely, they can also present retroperitoneally. Upadhyay et al reported a case of retroperitoneal enteric duplication cyst mimicking a pancreatic cyst [5].

\section{Synonyms}

Often a variety of terms are used depending upon the location like enteric cysts, enterogenous cyst, ileum duplex, giant diverticula, "unusual Meckel's diverticulum", accessory enteric formation etc... All these are covered under the generic term intestinal duplication.

\section{Structure}

The size varies from a few millimetres to more than a metre. Mucosa is type of mucosa of some part of the alimentary tract. Sometimes it is different from the adjacent part of the gut. If gastric ectopic mucosa is present, then acid secre- tion from this mucosa can result in peptic ulcer, perforation or bleeding. In one study [4], ectopic gastric mucosa was found in 21 duplications and pancreatic tissue was found in five (out of 101). True duplication has all the layers of the parent gut. Part of the muscular layer may be in continuity with the normal intestine.

\section{Incidence}

It is difficult to estimate the real incidence as many of them may be asymptomatic. It estimated to occur in about 1 in 5000 live births. Over 37 years, 101 duplications are seen in 96 patients in a major children's hospital [4]. From this it appears to be quite rare to see many cases in clinical practice. Seventy-five of these duplications were cystic and 26 were tubular.

\section{Clinical features}

It may be an incidental finding on routine $\mathrm{X}$ ray or ultrasound examination or at laparotomy. They usually present in the paediatric age group especially in the first two years of age. It is difficult to enumerate all possible variations as they are plenty and every possible combination is found. Presentation may be due to ectopic gastric mucosa secreting acid causing peptic ulceration, development of volvulus or gangrene, intestinal obstruction, intussusception, perforation, bleeding or asymptomatic palpable mass. Thus, the symptoms may be abdominal pain, vomiting, haematochezia or mass per abdomen. Duplication cysts in retrorectal area may mimic sacrococcegeal teratoma or anterior meningocoele.

Kibayashi et al [6] have reported a case of duplication which was presenting as diarrhoea and confused and treated as gastroenteritis in an 11 months old baby. The baby died within 24 hours. The correct diagnosis was established only at autopsy. There was a cyst of $3 \times 3 \times 4 \mathrm{~cm}$ size at mesenteric side of the terminal ileum, causing obstruction. Colonic duplications may present at a later age (5-12 years) with abdominal pain distension and constipation [7]. Volvulus was reported in $23.8 \%$ of the infants with duplications [8]. Acute intussusception was reported in $10.9 \%$ of paediatric cases. Rao et al reported a case of massive lower GI bleeding in 8 months old boy due to noncontiguous gastric duplication cyst in the pancreas. It had perforated into a loop of jejunum due to acid secretion from the gastric mucosa [9]. Mohnovski et al also reported a case of massive lower GI bleeding fallowing perforation of gastric duplication in to transverse colon and fistulisation [10]. Arbell et al reported a similar case of massive GI bleeding in 5 months old girl due to duodenal duplication cyst [11]. In both the cases the cyst was excised successfully after initial resuscitation. 
Martins et al [12] reported an interesting case of abdominal mass which disappeared after a few weeks. It reappeared after 1 year. Patient also developed acute intussusception. At laparotomy there was Ileo-caeco-colic intussusception and cystic caecal duplication of $4 \times 3 \mathrm{~cm}$ size with a hole. Duplication of appendix may present at later ages or may be asymptomatic. Usually they are found at appendicectomy.

de Tullio et al have reported adeno carcinomatous change in the enteric duplication in an 88 years old female patient attached to ileum [13].

\section{Associations}

A range of anomalies have been reported like vertebral anomalies like anterior spina bifida, missing vertebra, even cardiac anomalies. Colonic duplications may be associated with imperforate anus and or rectovaginal fistula [7]. Sarin [1] reported association of colonic triplication and urinary tract duplication with double bladder, diphallus and double urethra. One of the colons was ending in cloaca, second to normal anus and the third one ending as atresia.

\section{Investigations and diagnosis}

Plain X ray abdomen in erect posture may reveal gas under diaphragm or features of intestinal obstruction or peritonitis. Sometimes a radiolucent area representing cyst may be seen. Various imaging modalities like ultrasound, CT scan may help in imaging the cysts. Prada et al have reported right paraduodenal hernia in association with enteric duplication cyst diagnosed with contrast enhanced CT scan [14]. Ectopic gastric mucosa may be demonstrated by technetium 99 radionuclide scan. This scan is useful in children presenting with lower GI bleeding where Meckel's diverticulum is suspected. In the differential diagnosis intestinal duplication with gastric rests to be considered [15]. Barium enema helps in colonic duplication [9]. Voiding cysto urethrogram is useful when communication between urinary tract and colonic duplication is suspected [1]. Even prenatal diagnosis with good ultrasound examination is possible. Puligandla reported that prenatal diagnosis was possible in $31 \%$ of cases (cases of after 1991) [8]. However, $25 \%$ of these patients remained asymptomatic postnatally. Recent addition to the armamentarium of investigations in the diagnosis of intestinal pathology is wireless capsule endoscopy. Toth et al [16] reported a case of chronic recurrent anaemia in a 32 years adult cause of which was not diagnosed after GI endoscopy, enteroclysis, push enteroscopy, barium enema, colonoscopy and Meckel's scan. Wireless capsule endoscopy demonstrated ileal ulceration with stricture. This prompted laparotomy and finding of an intestinal duplication with ulceration.
Diagnosis is usually confirmed after surgical excision by histopathology which reveals mucosa of some part of the gastrointestinal tract.

\section{Management}

Management depends upon the presentation. Perforation, volvulus, intussusception, gangrene or severe bleeding requires laparotomy. Resection of the duplication cyst along with the involved segment of the intestine and end to end anastomosis of the intestine is possible if associated with small intestine. Marsupialisation in to the lumen of the duodenum can be done for cystic duplications at duodenum because excision can be dangerous. In tubular duplications also excision has to be done whenever possible, unless the attached intestine is too long. In such cases, stripping of the entire mucosa leaving behind sero muscular layer intact may be done. However, it is essential to ensure all ectopic gastric rests are removed. Surgical correction of the associated anomaly should also to be considered at appropriate ages. For example, posterior sagittal anorectoplasty, closure of recto vaginal fistula etc... Sometimes a colostomy is required as preliminary procedure to definitive repair. In case of a long tubular duplication of colon opening of the common wall by a long enterotomy by a stapler is possible [8].

$\mathrm{Li}$ et al [17] reported excision of the duplication without resection of the intestine successfully in 14 cases without any complications (which included 6 tubular and 8 cystic varieties). They claim the success is due to vascular pattern they described (vide supra).

Asymptomatic cysts may be left alone and observed. In fact many of them may never manifest in the life time of the patients.

Mortality depends upon presentation and severity of the associated anomalies. One series reports a mortality of about 1\%. Out of 96 cases, 94 underwent surgical management for their duplications. One duplication was found at necropsy, and one patient was asymptomatic and did not undergo operation. A single death occurred in a 2-day-old infant who had intrauterine volvulus and meconium peritonitis [4].

\section{Conclusion}

Intestinal duplications are rare congenital anomalies presenting clinically most commonly in first 2 years of age. They may be associated with other congenital anomalies like vertebral defects, congenital heart disease, anorectal anomalies etc... Ultrasound and CT scan are helpful in imaging the cyst. Technetium 99 radionuclide scan helps identifying the gastric rests. Diagnosis is established in most cases at laparotomy and is confirmed by histopathology by demonstrating mucosa corresponding to some part 
of the intestine. Morphologically they are classified into cystic and tubular types. Cystic variety is commoner and usually occurs near ileum.

Surgical management includes excision along with a segment of intestine and end to end anastomosis and, marsupialisation, opening of the common wall or observation in some asymptomatic cases etc... According to Li et al, excision is possible without resection of the intestinal segment.

\section{References}

1. Sarin YK, Manchanda V, Sharma A, Singhal A (2006) Triplication of colon with diphallus and complete duplication of bladder and urethra. J Pediatr Surg 41:1924-1926

2. Bremer JL (1944) Diverticula and duplications of the intestinal tract. Arch Pathol 38:132-140

3. Long Li, Zhang Jin-Zhe, Wang Yan Xia (1998) Vascular classification for small intestinal duplications. Experience with 80 cases. J Pediatr Surg 33:1243-1245

4. Holcomb GW, Gheissari A, O'Neill JA Jr, Shorter NA, Bishop HC (1989) Surgical management of alimentary tract duplications. Ann Surg 209(2):167-174

5. Upadhyay N, Gomez D, Button MF et al (2006) Retroperitoneal enteric duplication cyst presenting as a pancreatic cystic lesion. A case report. J Pancreas 7(5):492-495

6. Kibayashi K, Sumida T, Shojo H, Tokunaga O (2006) Unexpected death due to intestinal obstruction by a duplication cyst in an infant. Foresnic Science International 173: $175-177$

7. Blickman JG, Rieu PHM, Buonomo C, Hoogeveen YL, Boetes C (2006) Colonic duplications: Clinical presentations and radiological features in five cases. Europian $\mathrm{J}$ Radiol 59:14-19
8. Puligandla PS, Nguyen LT, St Vil, Flagoele, Bensoussan AL, Nguyen GH et al (2003) Gastrointestinal duplications. J Pediatr Surg 38:5

9. Rao KLN, Indira S, Ashwin P, Kim V, Sujith C (2003) Intrapancreatic gastric duplication cyst presenting as lower gastrointestinal bleeding. J Pediatr Surg 38:2: 243-244

10. Mahnovski V, Mahour G, Rowland J (1998) Gastric duplication-Colonic fistula with colonic ulceration and bleeding. J Pediatr Surg 33:1815-1816

11. Arbell D, Lebenthal A, Blashar A, et al (2002) Duplication cyst of duodenum as unusual cause of massive gastrointestinal bleeding in an infant. J Peditr Surg 37:5

12. Martins JL, Cury EK, Petrilli AS et al (2001) Cecal duplication causing a disappearing abdominal mass in an infant. $\mathrm{J}$ Pediatr Surg 36(10):1581-1583

13. Tullio D, Rinaldi R, Pellegrini D, et al (2008) Adenocarcinoma arising in an elderly patient's large ileal duplication. Int J Surg Pathol doi: 10.1177/1066896908319443

14. Marcos PA, Sanchis-Solera L, Perez C, Weihoff-Neuman A, Alonso-Jemenez L, Beltra-Pico R (2007) Computer tomography diagnosis of right paraduodenal hernia associated with enteric duplication cyst. J Pediatr Surg 42: 1938-1941

15. Eman KA and Mahmoud AA (2008) Intestinal duplication mimicking Meckel's diverticulum. Clin Nucl Med 33: 189-190

16. Toth E, Lillienau J, Ekelund J (2006) Ulcerated small-intestine duplication cyst: an unusual source of GI bleeding revealed by wireless capsule endoscopy. Gastrointestinal Endoscopy 63:192-194

17. Li L, Zhang J-Z, Chen J-J, Wang YX (1997) Conservative approach to duplication of the small bowel. J Pediatr Surg $32: 1679-1682$ 